\title{
Bipolar depression and treatment with antidepressants ${ }^{\dagger}$
}

Guy M. Goodwin

\section{Summary}

Treatment of bipolar disorder with antidepressants tested almost exclusively in unipolar cases is common but unsupported by an appropriate body of evidence. This anomaly is highlighted by a large Taiwanese study, which implies that patients with depression difficult to treat with antidepressants are quite likely to be diagnosed subsequently with bipolar disorder.

\section{Declaration of interest}

G.M.G. has held grants from Sanofi-Aventis and Servier, received honoraria for speaking or chairing educational meetings from AstraZeneca, Bristol-Myers Squibb, Eisai, Lundbeck, Sanofi-Aventis and Servier, and advised AstraZeneca, Boehringer-Ingelheim, Bristol-Myers Squibb, Janssen-Cilag, Lilly, Lundbeck, P1vital, Roche, Sanofi-Aventis, Servier and Wyeth. He holds shares in P1vital and acted as expert witness for Lilly and Servier.
Guy Goodwin is Professor of Psychiatry at the University of Oxford. His research interests are bipolar disorder and application of neuroscience in understanding its treatment.

The diagnosis and treatment of bipolar depression is controversial. Major depressive episodes occur in illness courses which may show severe, mild, minor or no mood elevation. These disorders are thus categorically bipolar I, bipolar II, bipolar spectrum and unipolar. The consensus around these diagnoses and an appreciation that there is greater comorbidity attached to those showing mood elevation is still quite recent. ${ }^{1,2}$ From the point of view of licensing authorities, an episode of major depression remains the same whether or not it occurs in the context of a bipolar illness course. Treatment of bipolar disorder with antidepressants tested almost exclusively in unipolar cases is therefore both common and sanctioned implicitly by product licenses. However, there is less confidence on the part of clinicians that the use of antidepressants can safely be generalised from unipolar cases to major depressive episodes in a bipolar illness course.

In the USA in particular, there has been concern that antidepressants will destabilise the illness and produce either mood instability or frank hypomanic or manic episodes when given to patients with bipolar depression. These reservations are clearly expressed in guidelines. ${ }^{3}$ This might appear to be a straightforward question of fact, but it is less simple than observing, from time to time, that patients taking antidepressants will switch into mania. Although all clinicians will have seen this occur, cause and effect cannot be automatically inferred. Such a switch is, after all, not necessarily surprising in someone who is at risk of manic episodes. Furthermore, it may be a necessary condition that it occurs from a euthymic state, which may have been restored by treatment with an antidepressant. We really require controlled studies where the effect of monotherapy with an antidepressant can be quantitatively compared with a mood stabiliser (or placebo). There are few such studies and almost all are short term (6-8 weeks). Tricyclic or dual-action antidepressants such as duloxetine or venlafaxine appear to be associated with short-term switches. ${ }^{4}$ Whether a bipolar illness can be induced by antidepressants or the longitudinal course made worse by antidepressants is unknown, even if that conclusion has often been drawn.

'See pp. 45-51, this issue.
A rather different perspective is expressed in doubts that antidepressants are at all efficacious in treating bipolar depression. It may seem strange to hold that they are simultaneously lacking in efficacy but also possessing the potential to induce rapid cycling and other adverse effects. The recent negative STEP-BD study, which compared mood stabiliser and antidepressant $v$. mood stabiliser and placebo therapy, has been accorded unjustified credibility in respect to lack of efficacy. ${ }^{5}$ It can just as plausibly be seen as a failed study that may have lacked assay sensitivity; moreover, there was no evidence for increased rates of switching to mania. It remains true, nevertheless, that the number of studies of bipolar depression is disappointing, even if the evidence still supports some superiority of antidepressants over placebo. ${ }^{4,6}$ Many psychiatrists in Europe remain happy to prescribe antidepressants for bipolar depression, and this difference from North American practice will only be resolved by improved evidence of the relative benefits and risks of antidepressants and the alternatives such as lamotrigine and quetiapine.

The publication by Li et $a l^{7}$ is of considerable interest because it offers a large-scale examination of the associations between antidepressant use in episodes of major depression and emergent bipolar disorder, using a nationwide database in Taiwan. The findings show that patients who require two or more changes of antidepressant (i.e. those whose depression is more difficult to treat) have a substantially increased probability of receiving a revised diagnosis of bipolar disorder. Over 7 years, 25\% of these patients received a bipolar disorder diagnosis, compared with rates below $10 \%$ in the patients who were treated with a single antidepressant and were deemed 'easy to treat'. This conclusively supports a widely held clinical belief that unrecognised bipolar disorder is a relatively important contributor to treatmentresistant depression. The hypothesis that bipolar depression may be less responsive to first-line antidepressants than unipolar depression would explain the findings.

As with other observational studies, it is difficult to decide whether the subsequent appearance of manic symptoms is a simple expression of a bipolar diathesis or should be attributed to the use of second- and third-line tricyclic antidepressants or other dual-action antidepressants. The data do not show why precisely the diagnosis was changed in any of these cases and we do not have documented the pattern or the number of manic symptoms.

Major depression is often the first presentation of bipolar I and bipolar II disorder and it would obviously be an advantage if we could predict from the beginning whether an individual 
has bipolar depression before the appearance of hypomania or mania. The consensus is that this is not possible other than on a probabilistic basis. ${ }^{8}$ Thus, in bipolar depression, patients are more likely to manifest objective psychomotor slowing, pathological guilt and an increased frequency of atypical symptoms such as hypersomnia and past psychotic depressive episodes. However, composite scores fail to identify even a point of rarity between the distributions of bipolar and unipolar depression. Although this is unhelpful to categorical diagnosis, it reinforces the increasingly accepted conclusion that a dimensional rather than a categorical approach across the spectrum from unipolar through to bipolar disorder is appropriate. ${ }^{1,6}$ In general, it appears to be broadly true that the more severe a depressive episode the more likely a subsequent bipolar diagnosis becomes, and of course perhaps the more difficult such cases may be to treat.

The take-home message for clinicians is that where patients, particularly young patients, have a pattern of illness that is suggestive of bipolarity (marked retardation, etc.) and they fail to respond to a first-line antidepressant, the possibility that they are likely to have the bipolar illness course increases and should be included in their prospective management.

Guy M. Goodwin, FMedSci, Oxford University Department of Psychiatry, Warneford Hospital, Oxford OX3 7JX, UK. Email: guy.goodwin@psych.ox.ac.uk

First received 17 Jul 2011, final revision 19 Oct 2011, accepted 26 Oct 2011

\section{References}

1 Angst J. The bipolar spectrum. Br J Psychiatry 2007; 190: 189-91.

2 Angst J, Cui L, Swendsen J, Rothen S, Cravchik A, Kessler RC, et al. Major depressive disorder with subthreshold bipolarity in the National Comorbidity Survey Replication. Am J Psychiatry 2010; 167: 1194-201.

3 Nivoli AMA, Colom F, Murru A, Pacchiarotti I, Castro-Loli P, González-Pinto A, et al. New treatment guidelines for acute bipolar depression: a systematic review. J Affect Disord 2011; 129: 314-26.

4 Gijsman HJ, Geddes JR, Rendell JM, Nolen WA, Goodwin GM. Antidepressants for bipolar depression: a systematic review of randomised controlled trials. Am J Psychiatry 2004; 161: 1537-47.

5 Sachs GS, Nierenberg AA, Calabrese JR, Marangell LB, Wisniewski SR, Gyulai $L$, et al. Effectiveness of adjunctive antidepressant treatment for bipolar depression. N Engl J Med 2007; 356: 1711-22.

6 Goodwin GM. Evidence-based guidelines for treating bipolar disorder: revised second edition - recommendations from the British Association for Psychopharmacology. J Psychopharmacol 2009; 23: 346-88.

7 Li C-T, Bai Y-M, Huang Y-L, Chen Y-S, Chen T-J, Cheng J-Y, et al. Association between antidepressant resistance in unipolar depression and subsequent bipolar disorder: cohort study. Br J Psychiatry 2012; 200: $45-51$.

8 Mitchell PB, Goodwin GM, Johnson GF, Hirschfeld RMA. Diagnostic guidelines for bipolar depression: a probabilistic approach. Bipolar Disord 2008; 10 $144-52$. 